\title{
SIMULTANEOUS OBSERVATION OF ENERGY AND ENSTROPHY CASCADES IN THIN-LAYER TURBULENCE
}

\author{
HUA XIA \\ Research School of Physics and Engineering, The Australian National University, Canberra ACT 0200, \\ Australia \\ hua.xia@anu.edu.au \\ NICOLAS FRANCOIS \\ Research School of Physics and Engineering, The Australian National University, Canberra ACT 0200, \\ Australia \\ nicolasfrancois2002@yahoo.fr \\ HORST PUNZMANN \\ Research School of Physics and Engineering, The Australian National University, Canberra ACT 0200, \\ Australia \\ horst.punzmann@anu.edu.au \\ DAVID BYRNE \\ ETH Zürich, Inst.f.Biogeochemie u.Schadstoffdynamik \\ Universitätstrasse 16 \\ 8092 Zuerich \\ david.byrne@usys.ethz.ch

\section{MICHAEL SHATS} \\ Research School of Physics and Engineering, The Australian National University, Canberra ACT 0200, \\ Australia \\ michael.shats@anu.edu.au \\ Published 22 June 2016
}

\begin{abstract}
We report the simultaneous observation of the inverse energy and direct enstrophy cascade in thinlayer turbulence. The experiments are conducted in an electromagnetically driven flow with layers of stratified fluid. Recent questions regarding the two-dimensionality of electromagnetically driven turbulence in such experiments are addressed.
\end{abstract}

Keywords: 2D turbulence; inverse cascade; enstrophy cascade.

\section{Introduction}

Electromagnetically driven turbulence in thin layers of fluids has been the focus of intense research for the past two decades. The question of whether such experiments are

This is an Open Access article published by World Scientific Publishing Company. It is distributed under the terms of the Creative Commons Attribution 3.0 (CC-BY) License. Further distribution of this work is permitted, provided the original work is properly cited. 
indeed two-dimensional still appears to be unsettled. Some doubts have arisen due to lack of simultaneous observation of both the inverse energy and forward enstrophy cascades as predicted ${ }^{1}$.

In conjunction, recent work ${ }^{2}$ reports results obtained in a single electrolyte layer. The paper presents stereo particle image velocimetry measurements of an electromagnetically driven dipole flow which displays a three-dimensional nature.

The purpose of this paper is to address the question of the two-dimensionality of thinfluid-layer experiments and discuss the conditions in which they can be treated as twodimensional.

The first such experiments ${ }^{3}$ were conducted with a layer of mercury on a solid substrate. This initial experiment supported the prediction by Kraichnan of the inverse energy cascade with the observation of $k^{-5 / 3}$ for half a decade of wave numbers below the energy injection scale.

The experiment was further improved by introducing stratified layers ${ }^{4}$. In their experiment, two $\mathrm{NaCl}$ solutions of different density were used. The flow was forced electromagnetically with a series of current pulses of opposite sign, with the upper fluid being driven by the fluid beneath. Using this arrangement to force at small scales, it confirmed Sommeria's results and observed energy spectra, $E(k) \sim k^{-5 / 3}$ over half a decade of wave numbers consistent with the inverse energy cascade ${ }^{5}$.

At this time, some of the questions regarding the two-dimensionality of thin-twolayer fluid experiments were addressed ${ }^{6}$. The authors concluded that after a short transient time the flow could be treated as two-dimensional. This work offered the first basis for assessing the two-dimensionality of thin fluid flows and was further given supporting evidence from numerical simulations ${ }^{7}$.

Later, using a different magnet $\operatorname{array}^{8}$, it is reported, for the first time in thin layers, energy spectra that was consistent with the enstrophy cascade $k^{-3}$ over a full decade of wave numbers. It should be noted here, that the magnetic field in this experiment was produced by randomly oriented magnets rather than with a regular checker-board like magnet array. We will show below that such randomness can lead to a broadening of the forcing in $k$-space. This can reduce the width of the inertial range, defined as a range with no forcing and dissipation. In fact, not only the lack of regularity in $\boldsymbol{B}$, but also a finiteness of the number of magnets broadens $k$-spectrum of forcing as will be shown later.

Layer stratification was further improved by using a Fluorinert (dielectric liquid) as the bottom layer ${ }^{9}$. The advantage of this setup is that there is no mixing of the two layers. In addition, since the dielectric fluid is non-conducting, forcing is confined to the top electrolyte layer only and forcing is thus detached from the bottom boundary layer.

In this paper we describe a thin-layer experiment that takes advantage of recent technological developments ${ }^{10-14}$. Using this experimental setup, we explore the twodimensionality of the flow and we observe simultaneously two inertial intervals. 


\section{Two-dimensional, thin-fluid-layer experiment}

The experimental setup is depicted in Figures 1. The fluid cell is a $300 \mathrm{x} 300 \mathrm{~mm}^{2}$ container made of Perspex. The bottom layer is a Fluorinert liquid which is nonconducting, chemically stable, nonflammable and has a specific gravity of $\mathrm{sg}=1.78$. The top layer is an electrolyte, $\mathrm{NaCl}$ solution, with a specific gravity of $\mathrm{sg}=1.03$. By varying the thicknesses of the layers, one can control the damping. The damping rate, $\alpha$ is estimated by measuring the decay rate of kinetic energy, $E_{t}=E_{t_{n}} e^{-\alpha\left(t-t_{0}\right)}$ after the forcing is ceased at some time $t_{0}$. By varying the layer thickness we obtain a range of values for $\alpha=0.05-0.5 \mathrm{~s}^{-1}$. In the experiments, the system size $L$ is varied by inserting boundary boxes of different sizes.

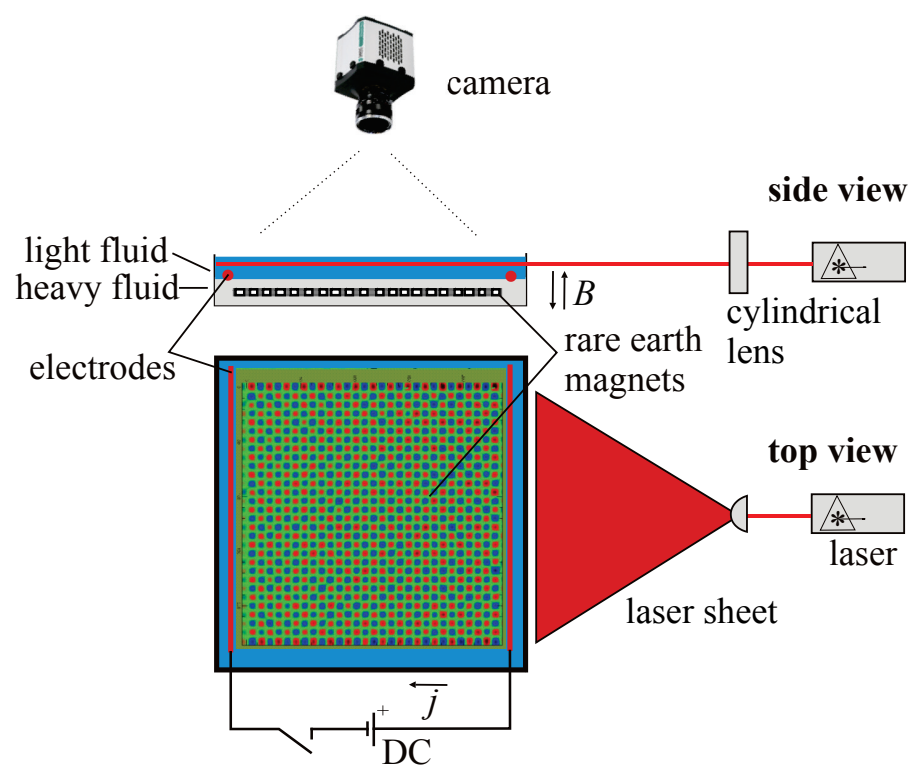

Fig. 1. Experimental setup for the electromagnetically driven turbulece.

An array of $24 \times 24$ neodynium-iron-boron (10 $\mathrm{mm}$ x $5 \mathrm{~mm} \times 4 \mathrm{~mm})$ permanent magnet dipoles with a separation of $10 \mathrm{~mm}$ centre to centre are submerged in the Fluorinert liquid. An electrical current $\mathbf{J}$, is driven through the electrolyte using two carbon electrodes. This results in the generation of $576 \mathbf{J X B}$ forced vortices which drive turbulence. We use modest forcing to avoid surface ripple and this is carefully monitored using laser reflection off the free surface.

The flow is visualised using neutrally buoyant $\mathrm{sg}=1.03$, polyamid seeding particles $50 \mu \mathrm{m}$ in diameter. These are illuminated by laser sheets of $1 \mathrm{~mm}$ thickness, produced by a $100 \mathrm{~mW}$ diode pumped solid state (DPSS) laser. The scattered light from the particles is captured using an Andor Neo sCMOS camera for the particle image velocimetry analysis. 


\subsection{Electromagnetic forcing}

The magnetic field $\mathbf{B}$ of the magnet array was characterised using a $2 \mathrm{D}$ scanning Hall probe. Two magnet arrays of different size, $24 \times 24(10 \mathrm{~mm} \times 5 \mathrm{~mm} \times 4 \mathrm{~mm})$ and $6 \times 6$ (25 mm x $10 \mathrm{~mm} \times 30 \mathrm{~mm}$ ), were compared prior to the experiments.

Figure 2 shows the contour plot of the vertical component of $\mathbf{B}$ for the $24 \times 24$ magnet array. Light/Dark (red/blue) contours depict upward/downward magnetic field and white is where $\mathbf{B}=0$.

Figure 3 and 4 show power spectra for the $24 \times 24$ and $6 \times 6$ magnet set respectively. The larger $24 \times 24$ number of magnets produces a spectrally narrow forcing scale almost two orders of magnitude above broadband forcing. The $6 \times 6$ magnet set has a much broader forcing scale and there exists harmonics above the forcing wave number.

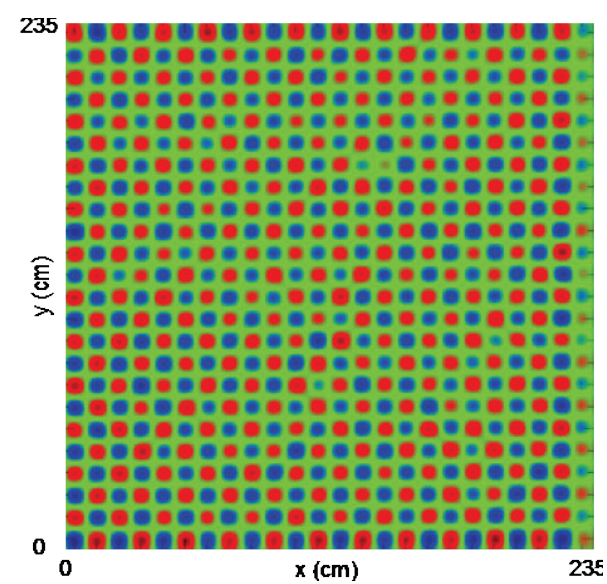

Fig. 2. Measurement of $B_{z}$ field of $24 \times 24 \$$ magnet matrix characterised by Hall probe measurements. Light/Dark (red/blue) contours depict upward/downward magnetic field and white (green) is where $\mathrm{B}=0$. Magnets are spaced equidistant $10 \mathrm{~mm}$ centre to centre. 


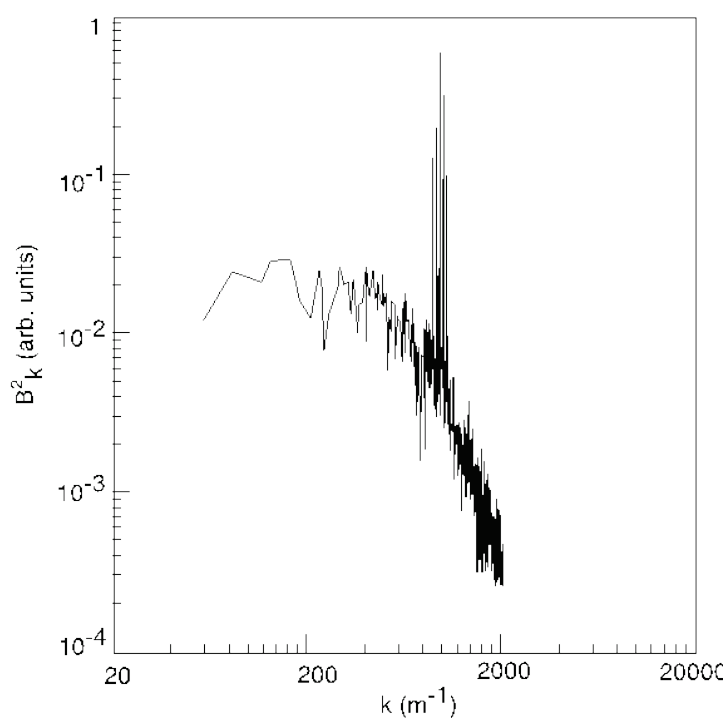

Fig. 3. Power spectrum of the magnetic field for the $24 \times 24$ magnet array. Larger number of magnets produces a spectrally narrow forcing scale.

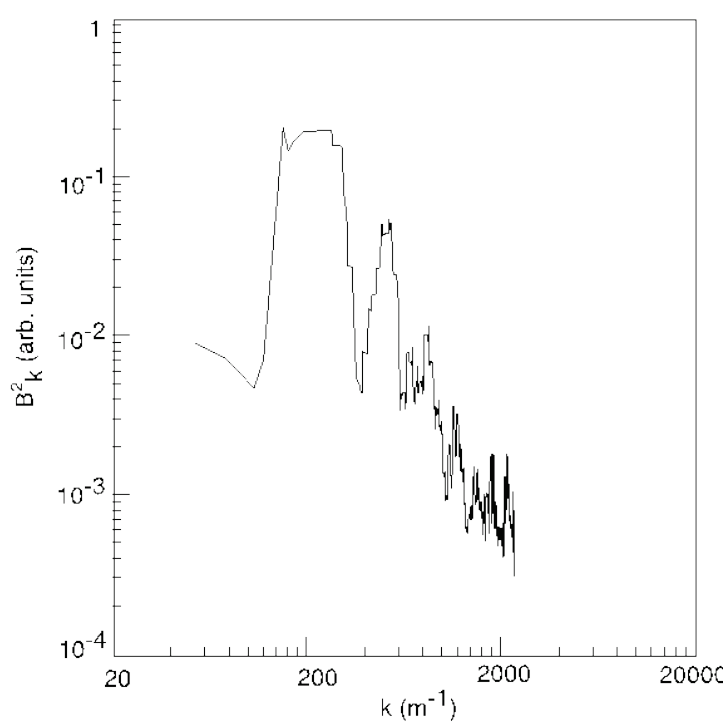

Fig. 4. Power spectrum of the magnetic field for the $6 \times 6$ magnet set. Less magnets with larger size produce broader forcing scale and there also exists harmonics above the forcing wave number. 


\subsection{Two-dimensionality}

To investigate the two-dimensionality of the flow, we perform two experiments. The first experiment is conducted in a single $10 \mathrm{~mm}$ layer of $\mathrm{NaCl}$ water solution. In a separate experiment, two $5 \mathrm{~mm}$ layers of Fluorinert and a $\mathrm{NaCl}$, water solution are used. The particle trajectories recorded during a $10 \mathrm{~s}$ long exposure are shown in Figure 5 for both experiments.

Figure 5 (a) shows the result for the two layer case. The particle streak lengths in this case are in excess of $100 \mathrm{~mm}$ and stop when the we stop recording. With a laser sheet thickness of $1 \mathrm{~mm}$, this equates to a vertical velocity component, $V_{z}$, of less than $1 \%$ of the horizontal velocity, $\left(V_{x}, V_{y}\right)$. The majority of particle streak lengths in Figure $5(\mathrm{~b})$, the single layer case, are $\sim 3 \mathrm{~mm}$ with a maximum length $\sim 10 \mathrm{~mm}$ before they disappear. This gives an estimate of the vertical velocity component of $\sim 10-30 \%$ of the horizontal velocity, in agreement with the results reported in Ref. 2.

Thus we conclude that stratification is essential for ensuring the two-dimensionality of the flow. Also, the detachment of forcing from the solid bottom helps reduce the effect of the bottom boundary layer on the bulk flow.

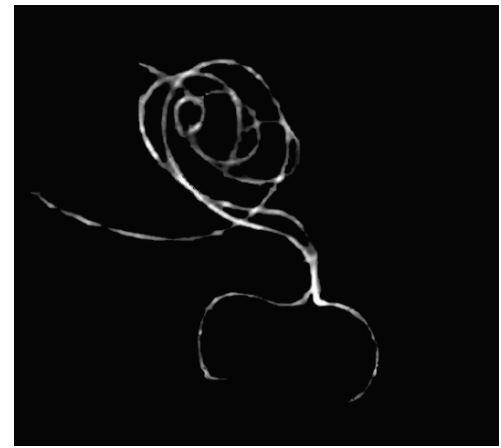

(a)

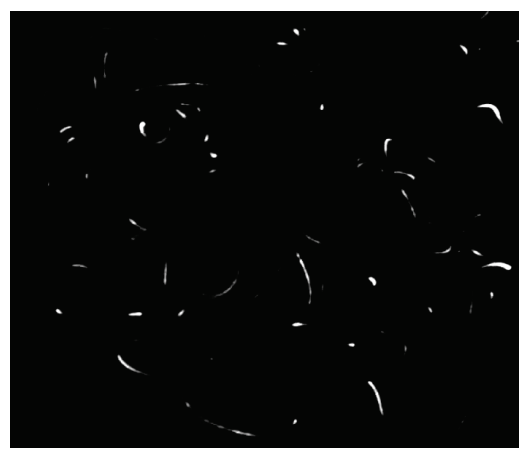

(b)

Fig. 5. Particle trajectories from a single $\$ 10 \$ \mathrm{~s}$ long exposure. a) Two fluid layers both with $\$ 5 \$ \mathrm{~mm}$ thicknesses. b) Single fluid layer $\$ 10 \$ \mathrm{~mm}$ thickness.

\section{Results}

Typically, the flow is recorded for 600 frames or $200 \mathrm{~s}$ with both the initial stage and decay stage lasting approximately 30 seconds. The flow is forced with a DC current of 1.0-1.5 A. 


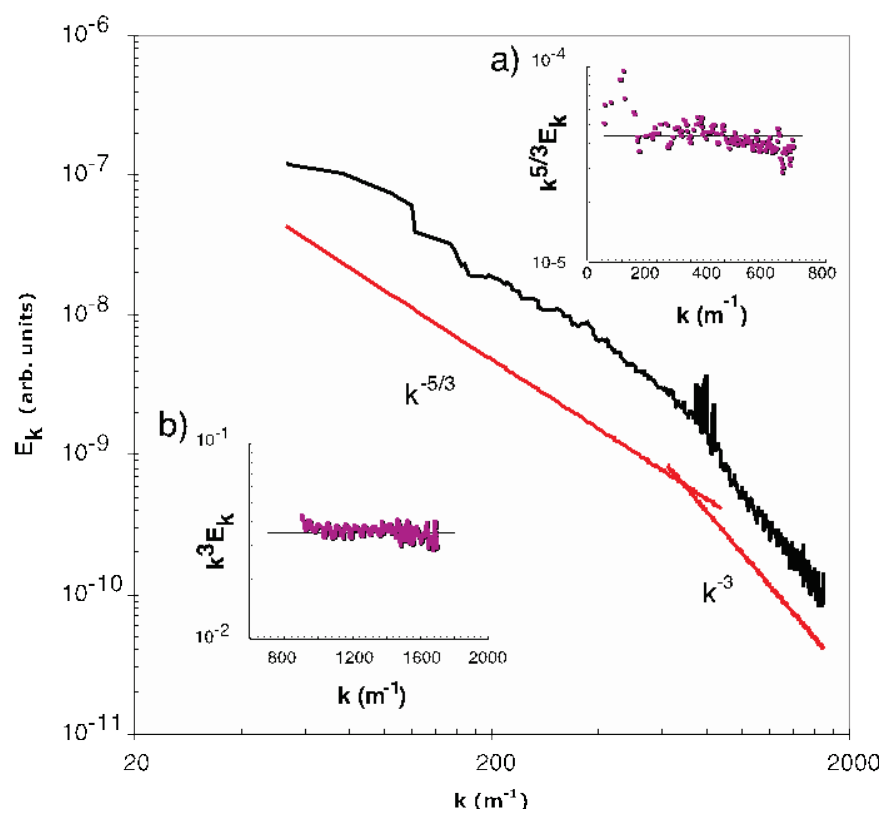

Fig. 6. Total energy spectrum for $235 \mathrm{~mm}$ boundary, averaged over 300 realisations. Inset a) shows spectrum re-scaled with scaling coefficient $k^{5 / 3} \mathrm{E}(\mathrm{k})$. Inset b) $k^{3} \mathrm{E}(\mathrm{k})$.

The resulting computed energy spectra is shown in Figure 6 with Fluorinert and $\mathrm{NaCl}$ solution layer thicknesses of $3 \mathrm{~mm}$ and $4 \mathrm{~mm}$ respectively. The flow was confined to a region $(235 \times 235 \mathrm{~mm})$. The damping rate was measured to be $\sim 0.3 \mathrm{~s}^{-1}$. The spectrum is averaged over 300 realisations and shows, for the first time in a thin-fluid experiment, simultaneously $k^{-5 / 3}$ inverse energy cascade and $k^{-3}$ direct enstrophy cascade over almost a decade and half a decade of wave numbers respectively. The good agreement with both forward and enstrophy cascade scaling is shown in inset a) and b) by re-scaling the two ranges by their scaling coefficient.

Due to the bounding box the flow is not ideally isotropic and wave numbers $k<150$ $\mathrm{m}^{-1}$ shows some anisotropy in the flow. This long lived structure does not follow the $\mathrm{k}^{-5 / 3}$ scaling. This is shown in figure 6 inset a) and can also be seen in the two-dimensional power spectrum, Figure 7. Here, the four grey dots represent the forcing scale. One can see that in the inertial ranges in both directions (where toward centre represents large scales) isotropy is preserved except for large scales.

In summary, it has been shown and confirmed in this paper, that there are conditions under which fluid-layer experiments may exhibit three-dimensional flow components. One must be mindful of these conditions to ensure the two-dimensionality of the flow. Our experiment incorporates aspects that we believe are pivotal to ensuring twodimensionality. First is stratification using two layers. This detaches the forcing from the bottom boundary and also controls damping. Second, the checker-board arrangement and use of a large number of forcing magnets to ensure a spectrally narrow forcing scale. Also, within the fluid-layer, one has to make sure that the dominant component of the 
magnetic field is vertical. Modest forcing is essential to avoid surface ripple and in our case this is monitored with laser beam reflection off the free surface.

The results obtained in our experiments show good agreement with two-dimensional turbulence theory resolving both the inverse and forward 2D turbulence cascades. This, in conjunction with a measured vertical velocity component of less than $1 \%$, provides solid evidence that the thin-fluid-layer laboratory experiment is an accurate model and testing ground for two-dimensional turbulence theory.

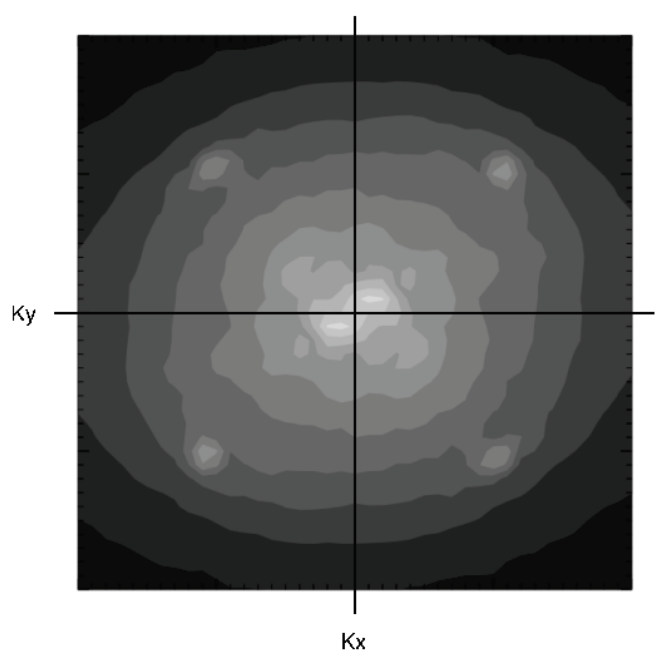

Fig. 7. Two-dimensional power spectrum. Four grey dots represent forcing scale. Isotropy is preserved except for large scales $\mathrm{k}<150 \mathrm{~m}^{-1}$ (centre of plot).

\section{Acknowledgments}

HX would like to acknowledge the support by the Australian Research Council's Future Fellowship (FT140100067).

\section{References}

1. R.H. Kraichnan. Intertial ranges in two-dimensional turbulence. Physics of Fluids, 10:1417-1423, 1967.

2. R. A. D. Akkermans, L. P. J. Kamp, H. J. H. Clercx, and G. J. F. van Heijst. Intrinsic three-dimensionality in electromagnetically driven shallow flows. Euro- physics Letters, 83:24001, 2008.

3. J. Sommeria. Experimental study of the two- dimensional inverse energy cascade in a square box. Journal of Fluid Mechanics, 170:139-168, 1986.

4. J. Paret and P. Tabeling. Experimental observation of the two-dimensional inverse energy cascade. Phys. Rev. Lett., 79(21):4162-4165, 1997.

5. J. Paret and P. Tabeling. Intermittency in the two- dimensional inverse cascade of energy: Experimental observations. Physics of Fluids, 10:3126-3136, 1998.

6. J. Paret, D. Marteau, O. Paireau, and P. Tabeling. Are flows electromagnetically forced in thin stratified lay- ers two dimensional? Physics of Fluids, 9(10):3102-3104, 1997. 
7. B. Juttner, D. Marteau, P. Tabeling, and A. Thess. Numerical simulations of experiments on quasi-two- dimensional turbulence. Phys. Rev. E, 55(5):5479-5488, 1997.

8. J. Paret, M. Jullien, and P. Tabeling. Vorticity statistics in the two-dimensional enstrophy cascade. Phys. Rev. Lett., 83(17):3418-3421, 1999.

9. M. K. Rivera and R. E. Ecke. Pair dispersion and doubling time statistics in twodimensional turbulence. Phys. Rev. Lett., 95:194503-1-4, 2005.

10. H. Xia, H. Punzmann, G. Falkovich, and M. G. Shats. Turbulence-condensate interaction in two dimensions. Phys. Rev. Lett., 101:194504, 2008.

11. H. Xia, M. Shats and G. Falkovich, Spectrally Condensed Turbulence in Thin Layers, Physics of Fluids, 21, 125101(2009)

12. D. Byrne, H. Xia and M. Shats, Robust inverse energy cascade and turbulence structure in three-dimensional layers of fluid, Physics of Fluids 23, 095109 (2011), doi:10.1063/1.3638620

13. H. Xia, D. Byrne, G. Falkovich and M. Shats, Upscale energy transfer in thick turbulent fluid layers, Nature Physics 7 321-324 (2011), doi:10.1038/nphys1910

14. M. Shats, D. Byrne and H. Xia, Turbulence decay rate as a measure of flow dimensionality, Physical Review Letters, 105, 264501 (2010) 